\title{
ON THE EXPONENTS OF IDEAL CLASS GROUPS OF CYCLOTOMIC FIELDS
}

\author{
KUNIAKI HORIE \\ (Communicated by William Adams)
}

\begin{abstract}
It will be proved that the ideal class group of the cyclotomic field of 65 th roots of unity is of type $(4,4,2,2)$, and remarks on the exponents of ideal class groups of cyclotomic fields will be made.
\end{abstract}

Let $\mathbb{Q}$ be the rational field, $\mathbb{Z}$ the additive group of (rational) integers, and $\mathbb{N}$ the set of positive integers. We shall assume all algebraic extensions over $\mathbb{Q}$ dealt with hereafter to be contained in the complex field. The exponent of each finite group $G$ will be denoted by $\exp G$. For any $m \in \mathbb{N}$, let $C_{m}$ denote the ideal class group of the cyclotomic field $\mathbb{Q}\left(e^{2 \pi i / m}\right)$. We then note that $\mathbb{Q}\left(e^{2 \pi i / m^{\prime}}\right)=\mathbb{Q}\left(e^{2 \pi i / m}\right)$ for some $m^{\prime} \in \mathbb{N}$ less than $m$ if and only if $m \equiv 2$ $(\bmod 4)$.

In this paper, we shall prove the following results.

Proposition 1. $C_{65}$ is isomorphic as an abelian group to the direct sum of two copies of $\mathbb{Z} / 4 \mathbb{Z}$ and two copies of $\mathbb{Z} / 2 \mathbb{Z}$ :

$$
C_{65} \cong(\mathbb{Z} / 4 \mathbb{Z})^{2} \oplus(\mathbb{Z} / 2 \mathbb{Z})^{2} .
$$

Proposition 2. Let $m$ be a positive integer $\not \equiv 2(\bmod 4)$. Then:

(i) $\exp C_{m}=2^{n}$ does not hold for any $n \in \mathbb{N}$ exceeding 3 ,

(ii) $\exp C_{m}=8$ is equivalent with $m=68$,

(iii) $\exp C_{m}=4$ is equivalent with $m=65$ or 120 ,

(iv) $\exp C_{m}=2$ is equivalent with $m=29,39$, or 56 .

Remark. It is well known that for $m$ in Proposition 2 , $\exp C_{m}=1$, i.e., $C_{m}=$ $\{1\}$ if and only if $m \in\{1,3,4,5,7,8,9,11,12,13,15,16,17,19,20,21$, $24,25,27,28,32,33,35,36,40,44,45,48,60,84\}$ (cf. [9]).

Let us prove Proposition 1. We put

$$
K=\mathbb{Q}(\zeta) \text { where } \zeta=e^{2 \pi i / 65} .
$$

Received by the editors April 2, 1992.

1991 Mathematics Subject Classification. Primary 11R18, 11R29; Secondary 11R27, 11R37.

Supported in part by Grant-in-Aid for Scientific Reserch (No. 04640066), Ministry of Education, Science and Culture, Japan. 
The class number of $K$ equals $2^{6}$ by $[2,6,8]:\left|C_{65}\right|=2^{6}$. For any finite abelian group $A$, let $r(A)$ denote the rank of $A$. We shall first show $r\left(C_{65}\right)=4$ and then show $r\left(C_{65}^{2}\right)=2$.

Let $H$ be the maximal unramified abelian extension over $K, k$ the maximal real subfield of $K$, and $L$ the maximal abelian extension over $k$ in which no finite prime of $k$ is ramified. Note that $k \subseteq K \subseteq L \subseteq H$. The integral group ring of $\mathrm{Gal}(K / k)$ acts on both $C_{65}$ and $\mathrm{Gal}(H / K)$ in the obvious manner. Let $L^{\prime}$ be the maximal unramified abelian extension over $K$ such that $\exp \operatorname{Gal}\left(L^{\prime} / K\right) \leq 2$, i.e., the intermediate field of $H / K$ such that $\operatorname{Gal}\left(H / L^{\prime}\right)=\operatorname{Gal}(H / K)^{2}$. Let $j$ be the complex conjugation of $K$, i.e., the generator of $\operatorname{Gal}(K / k):\langle j\rangle=\operatorname{Gal}(K / k)$. Since the class number of $k$ equals 1 (cf. [8]), we then have $C_{65}^{1+j}=\{1\}$ so that, by class field theory,

$$
\operatorname{Gal}(H / K)^{1-j}=\operatorname{Gal}(H / K)^{2}=\operatorname{Gal}\left(H / L^{\prime}\right) .
$$

Hence $L^{\prime}$ is a Galois extension over $k$ and

$$
\operatorname{Gal}\left(L^{\prime} / k\right)=\left\langle j^{\prime}\right\rangle \times \operatorname{Gal}\left(L^{\prime} / K\right)
$$

where $j^{\prime}$ is the complex conjugation of $L^{\prime}$. It therefore follows that $L^{\prime}$ is an abelian extension over $k$ in which no finite prime of $k$ is ramified. This fact means

$$
L^{\prime} \subseteq L \text {. }
$$

Now, let $E$ be the unit group of $K, E^{+}$the unit group of $k, k_{+}$the subgroup of the multiplicative group $k^{\times}$consisting of all totally positive numbers in $k, P$ the group of principal ideals of $k$, and $\mathscr{O}$ the ring of algebraic integers in $k$;

$$
P=\left\{\alpha \mathscr{O} \mid \alpha \in k^{\times}\right\} .
$$

Let $P_{+}$denote the subgroup of $P$ defined by

$$
P_{+}=\left\{\beta \mathscr{O} \mid \beta \in k_{+}\right\} \text {. }
$$

Then, letting each $\alpha \in k^{\times}$correspond to $\alpha \mathscr{O}$, we obtain homomorphisms

$$
k^{\times} \longrightarrow P, \quad k_{+} \longrightarrow P_{+} .
$$

These obviously induce an exact sequence

$$
\{1\} \longrightarrow E^{+} / E_{+} \longrightarrow k^{\times} / k_{+} \longrightarrow P / P_{+} \longrightarrow\{1\},
$$

where $E_{+}$denotes the group of totally positive units in $E^{+}: E_{+}=E^{+} \cap k_{+}$. As the class number of $k$ equals 1 , we obtain $\operatorname{Gal}(L / k) \cong P / P_{+}$. In particular, $L \subseteq L^{\prime}$ so that $L^{\prime}=L$ by $(1)$. Hence it follows from $k^{\times} / k_{+} \cong(\mathbb{Z} / 2 \mathbb{Z})^{[k: \mathbb{Q}]}$ that

$$
r\left(\operatorname{Gal}\left(L^{\prime} / K\right)\right)=r\left(P / P_{+}\right)-1=[k: \mathbb{Q}]-1-r\left(E^{+} / E_{+}\right) .
$$

Consequently,

$$
r\left(C_{65}\right)=23-r\left(E^{+} / E_{+}\right) .
$$

The main result of [10] (based on the analytic class number formula) implies that since just two distinct prime numbers are ramified in $K$ and since the class number of $k$ equals $1, E$ is contained in the subgroup of $K^{\times}$generated by $1-\zeta^{u}$ for all $u \in \mathbb{Z}$ with $65 \nmid u$, whence $E$ is generated by $-\zeta, 1-\zeta^{a}$ for all 
$a \in\{1, \ldots, 32\}$ prime to $65,\left(1-\zeta^{5 b}\right) /\left(1-\zeta^{5}\right)$ for all $b \in\{2,3,4,5,6\}$, and $\left(1-\zeta^{26}\right) /\left(1-\zeta^{13}\right)$ :

$$
E=\left\langle-\zeta, 1-\zeta^{a}, \frac{1-\zeta^{5 b}}{1-\zeta^{5}}, \frac{1-\zeta^{26}}{1-\zeta^{13}}\right\rangle_{1 \leq a \leq 32,(a, 65)=1 ; 2 \leq b \leq 6} .
$$

Let $E^{\prime}$ be the subgroup of $E^{+}$with generators $-1,(1-\zeta)\left(1-\zeta^{-1}\right)=|1-\zeta|^{2}$, $\sin \frac{2 \pi a}{65} / \sin \frac{2 \pi}{65}=\left(\zeta^{a}-\zeta^{-a}\right) /\left(\zeta-\zeta^{-1}\right)$ for all $a \in\{2, \ldots, 32\}$ prime to 65 , $\sin \frac{2 \pi b}{13} / \sin \frac{2 \pi}{13}$ for all $b \in\{2, \ldots, 6\}$, and $\sin \frac{4 \pi}{5} / \sin \frac{2 \pi}{5}$. Then

$$
(1-\zeta)^{2}=-\zeta(1-\zeta)\left(1-\zeta^{-1}\right) \in\langle-\zeta\rangle E^{\prime}, \quad E=\langle 1-\zeta,-\zeta\rangle E^{\prime}
$$

so the index $\left[E:\langle-\zeta\rangle E^{\prime}\right]$ does not exceed 2 . However, $\left[E:\langle-\zeta\rangle E^{+}\right]=2$ as is well known. Thus $\langle-\zeta\rangle E^{\prime}=\langle-\zeta\rangle E^{+}$. It is now easy to see $E^{\prime}=E^{+}$:

$$
E^{+}=\left\langle-1,|1-\zeta|^{2}, \frac{\sin \frac{2 \pi a}{65}}{\sin \frac{2 \pi}{65}}, \frac{\sin \frac{2 \pi b}{13}}{\sin \frac{2 \pi}{13}}, \frac{\sin \frac{4 \pi}{5}}{\sin \frac{2 \pi}{5}}\right\rangle_{2 \leq a \leq 32,(a, 65)=1 ; 2 \leq b \leq 6} .
$$

Next, for any $n \in \mathbb{N}$, let $n^{*}$ denote the number of distinct positive integers $\leq n$ prime to 65 . With $c$ varying through the positive integers $\leq 32$ prime to 65 , put

$$
\begin{aligned}
f\left(1, c^{*}\right) & =1+2 \mathbb{Z}, \\
f\left(a^{*}, c^{*}\right) & =\left[\frac{2 a c}{65}\right]+2 \mathbb{Z} \text { for } a \in\{2, \ldots, 32\},(a, 65)=1, \\
f\left(b+23, c^{*}\right) & =\left(\left[\frac{2 b c}{13}\right]-\left[\frac{2 c}{13}\right]\right)+2 \mathbb{Z} \text { for } b \in\{2, \ldots, 6\}, \\
f\left(30, c^{*}\right) & =\left(\left[\frac{4 c}{5}\right]-\left[\frac{2 c}{5}\right]\right)+2 \mathbb{Z} .
\end{aligned}
$$

Here $[q]$ denotes for each $q \in \mathbb{Q}$ the maximal integer $\leq q$ and we understand that, for each $u \in \mathbb{Z}, u+2 \mathbb{Z}=\{u+2 v \mid v \in \mathbb{Z}\}$ belongs to $\mathbb{F}_{2}$, the field whose additive group is $\mathbb{Z} / 2 \mathbb{Z}$. We can then define a matrix

$$
M=(f(s, t))_{1 \leq s \leq 30,1 \leq t \leq 24}
$$

of size $(30,24)$ with coefficients in $\mathbb{F}_{2}$. Furthermore, we readily see from (3) that $r\left(E^{+} / E_{+}\right)$equals the rank of $M$ while elementary calculations show that the rank of $M$ equals 19 . Therefore we obtain $r\left(C_{65}\right)=4$ from (2).

Now, let $F$ be the subfield of $K$ such that $[K: F]=3$, and let $\sigma$ be a generator of $\operatorname{Gal}(K / F)$. As the class number of $F$ equals 1 (cf. [2, 8]), we have

$$
C_{65}^{1+\sigma+\sigma^{2}}=\{1\}
$$

viewing $C_{65}$ as a module over the integral group ring of $\mathrm{Gal}(K / F)$. On the other hand, we have $r\left(C_{65}^{2}\right)=1$ or 2 by $\left|C_{65}\right|=2^{6}$ and $r\left(C_{65}\right)=4$. In particular, there exists an element $x$ of $C_{65}$ with $x^{2} \notin C_{65}^{4}$. If $r\left(C_{65}^{2}\right)=1$ or equivalently $C_{65}^{2} / C_{65}^{4} \cong \mathbb{Z} / 2 \mathbb{Z}$, then $x^{2 \sigma} C_{65}^{4}=x^{2} C_{65}^{4}$ so that

$$
x^{2} \in x^{6} C_{65}^{4}=x^{2\left(1+\sigma+\sigma^{2}\right)} C_{65}^{4} \text {. }
$$


This conclusion contradicts (4), however (for a general argument, cf. [12, Theorem 10.8]). Hence

$$
r\left(C_{65}^{2}\right)=2, \quad \text { namely, } C_{65} \cong(\mathbb{Z} / 4 \mathbb{Z})^{2} \oplus(\mathbb{Z} / 2 \mathbb{Z})^{2} .
$$

It is proved in [3] that if $m$ is a positive integer $\not \equiv 2(\bmod 4)$ different from $29,39,56,65,68,120$, then $\left|C_{m}\right|$ either equals 1 or has an odd prime divisor. Proposition 2 therefore follows from Proposition 1 and the well-known facts below (cf. $[1,4,5,8,11])$.

$$
C_{29} \cong(\mathbb{Z} / 2 \mathbb{Z})^{3}, \quad C_{39} \cong C_{56} \cong \mathbb{Z} / 2 \mathbb{Z}, \quad C_{68} \cong \mathbb{Z} / 8 \mathbb{Z}, \quad C_{120} \cong \mathbb{Z} / 4 \mathbb{Z} .
$$

Remark. Modifying the proof of Proposition 1, we can further find other facts such as

$$
C_{77} \cong(\mathbb{Z} / 4 \mathbb{Z})^{4} \oplus(\mathbb{Z} / 5 \mathbb{Z}), \quad C_{87} \cong(\mathbb{Z} / 8 \mathbb{Z})^{3} \oplus(\mathbb{Z} / 3 \mathbb{Z}), \quad C_{156} \cong \mathbb{Z} / 4 \cdot 3 \cdot 13 \mathbb{Z} ;
$$

but we omit the details here.

\section{ACKNOWLEDGMENT}

The author thanks his wife Mitsuko for helpful conversations.

\section{REFERENCES}

1. F. Gerth, The ideal class groups of two cyclotomic fields, Proc. Amer. Math. Soc. 78 (1980), 321-322.

2. H. Hasse, Über die Klassenzahl abelscher Zahlkörper, Akademie Verlag, Berlin, 1952; Springer-Verlag, Berlin, Heidelberg, New York, and Tokyo, 1985.

3. K. Horie, On the class numbers of cyclotomic fields, Manuscripta Math. 65 (1989), 465-477.

4. K. Iwasawa, A note on ideal class groups, Nagoya Math. J. 27 (1966), 239-247.

5. E. E. Kummer, Über die Irregularität von Determinanten, Monatsber. Akad. Wiss. Berlin (1853), 194-200; Collected Papers, I, 539-545.

6. _ Über die Klassenzahl der aus n-ten Einheitswurzeln gebildeten complexen Zahlen, Monatsber. Akad. Wiss. Berlin (1861), 1051-1053 ; Collected Papers, I, 883-885.

7. S. Louboutin, Détermination des corps quartiques cycliques totalement imaginaires à groupe des classes d'idéaux d'exposant $\leq 2$, Manuscripta Math. 77 (1992), 385-404.

8. J. M. Masley, Class numbers of real cyclic number fields with small conductor, Compositio Math. 37 (1978), 297-319.

9. J. M. Masley and H. L. Montgomery, Cyclotomic fields with unique factorization, J. Reine Angew. Math. 286/287 (1976), 248-256.

10. W. Sinnott, On the Stickelberger ideal and the circular units of a cyclotomic fiela', Ann. of Math. (2) 108 (1978), 107-134.

11. K. Tateyama, On the ideal class groups of some cyclotomic fields, Proc. Japan Acad. Ser. A 58 (1982), 333-335.

12. L. C. Washington, Introduction to cyclotomic fields, Springer Verlag, New York, Heidelberg, and Berlin, 1982.

Department of Mathematics, Nara Women's University, Kita-Uoya Nishimachi, Nara 630, JAPAN

Current address: Department of Mathematics, Tokai University, 1117 Kitakaname, Hiratsuka, Kanagawa 259-12, Japan 